FORMATION Formation emploi

Revue française de sciences sociales

103 | juillet-septembre 2008

Fuite ou mobilité des cerveaux?

\title{
Mobilité internationale des étudiants et débuts de vie active
}

International Mobility of Students and Early Career

Internationale Mobilität von Studenten und Early Career

Movilidad internacional de los estudiantes y comienzo de vida activa

\section{Harald Schomburg et Ulrich Teichler}

\section{(2) OpenEdition}

Journals

\section{Édition électronique}

URL : http://journals.openedition.org/formationemploi/2411

DOI : 10.4000/formationemploi.2411

ISSN : 2107-0946

\section{Éditeur}

La Documentation française

\section{Édition imprimée}

Date de publication : 1 septembre 2008

Pagination : 41-55

ISSN : 0759-6340

\section{Référence électronique}

Harald Schomburg et Ulrich Teichler, "Mobilité internationale des étudiants et débuts de vie active», Formation emploi [En ligne], 103 | juillet-septembre 2008, mis en ligne le 01 septembre 2010, consulté le 30 octobre 2020. URL : http://journals.openedition.org/formationemploi/2411 ; DOI : https:// doi.org/10.4000/formationemploi.2411 


\section{DOSSIER}

\section{Mobilité internationale des étudiants et débuts de vie active}

Par Harald Schomburg et Ulrich Teichler*

De plus en plus de diplômés ont une expérience internationale. Pourtant, de moins en moins d'anciens étudiants mobiles notent un réel impact de leurs expériences internationales sur leur carrières en général et sur la dimension internationale de leurs activités professionnelles en particulier.

La Déclaration de Bologne, signée en 1999 par près de trente ministres européens en charge de l'enseignement supérieur, préconise que la nouvelle structure baccalauréat-master, ainsi que les différentes mesures qui l'accompagnent sont essentielles pour rendre l'enseignement supérieur des pays européens plus attractif pour des étudiants du monde entier et pour favoriser la mobilité étudiante entre les pays européens, au cours de leurs études, mais également pour leur carrière professionnelle qui commence. $\mathrm{La}$ mobilité et l'acquisition de compétences nécessaires à la mobilité sont souvent considérées comme des facteurs importants pour les activités réalisées dans leur pays d'origine, puisque les compétences en langues étrangères, la connaissance des autres pays et la compréhension du monde international sont utiles. La mobilité à l'international et l'apprentissage international dans le pays d'origine peuvent également améliorer les compétences des étudiants et les performances professionnelles en général des diplômés, en renforçant par exemple leur maturité grâce à la prise en charge de tâches imprévues.
* Harald Schomburg est chercheur senior de l'International Centre for Higher Education Research (INCHERKassel), université de Kassel, Allemagne. Ses domaines de recherche portent principalement sur les relations entre l'éducation et l'emploi, les enquêtes auprès des diplômés et leur rôle dans le développement de l'enseignement supérieur ainsi que sur la mobilité internationale des étudiants et des chercheurs.

Ulrich Teichler est professeur à l'université de Kassel, Allemagne, et assure depuis seize ans les fonctions de directeur de l'International Centre for Higher Education Research lanciennement "Centre for Research on Higher Education and Work »). II a été expert auprès de l'OCDE pour les politiques d'éducation dans plusieurs pays. Ses domaines de recherche portent principalement sur les relations entre les systèmes de l'éducation et de l'emploi, les modèles institutionnels de l'enseignement supérieur, la politique en matière d'éducation et les conséquences des programmes de mobilité et de coopération internationales. II a publié de nombreux ouvrages et tenu diverses conférences sur ces sujets. 
Mais la mobilité internationale, et en particulier la mobilité temporaire au cours des études, donne-t-elle réellement des résultats positifs? La valeur professionnelle de la mobilité des étudiants a-t-elle été prouvée ? Nous présentons dans cet article les résultats d'études récentes réalisées à grande échelle et qui peuvent répondre en partie à ces interrogations. L'accent sera mis sur l'emploi et le travail des anciens étudiants mobiles pour définir l'impact de la mobilité étudiante et donc éventuellement l'impact du processus de Bologne en tant qu'outil envisagé pour la favoriser. Tout d'abord, nous décrivons brièvement les sources utilisées dans cet article. Ensuite, nous essayons de synthétiser les éléments disponibles sur l'ampleur de la mobilité étudiante. Puis nous procédons à une comparaison entre les pays, pour obtenir des réponses à la question centrale posée dans cet article : la mobilité internationale des étudiants a-t-elle des effets positifs sur leur carrière professionnelle?

\section{AMÉLIORER LA MOBILITÉ ÉTUDIANTE À L'INTERNATIONAL}

Par mobilité étudiante à l'international, on entend plus particulièrement la mobilité géographique des étudiants vers des pays étrangers. On distingue au moins deux groupes d'étudiants mobiles à l'international : a) les étudiants étrangers (au sens de leur nationalité ou par le fait qu'ils sont nés dans un pays étranger) participant à un programme d'études dans le but d'obtenir un diplôme ; b) les étudiants temporairement mobiles, qui se rendent à l'étranger pendant leurs études uniquement pour une courte période (comme le programme SOCRATES/ERASMUS).

Les statistiques publiées par EUROSTAT, l'OCDE (Organisation de coopération et de développement économiques) et l'UNESCO sur la mobilité étudiante s'appuient sur les données recueillies par chaque pays. Elles fournissent les informations sur les étudiants étrangers, lesquels ne représentent qu'une partie des étudiants mobiles. Cependant, le nombre actuel d'étudiants mobiles en Europe est différent du nombre d'étudiants étrangers représentés dans les statistiques d'éducation, dans trois domaines notamment (voir Kelo, Teichler et Wächter, 2006) :
- De nombreux étudiants étrangers ont déjà suivi une formation dans leur pays d'études avant l'enseignement supérieur. Ces étudiants n'apparaissent pas comme des étudiants mobiles au sens de la mobilité internationale pour les études dans un autre pays. Les statistiques d'éducation ont ainsi tendance à surestimer le nombre d'étudiants mobiles.

- Les étudiants qui suivent, temporairement, leurs études à l'étranger (comme les étudiants ERASMUS et d'autres programmes d'échanges), la plupart pour une durée d'une année ou d'un semestre, ne sont que partiellement ou pas du tout représentés dans les statistiques des étudiants étrangers de nombreux pays (les statistiques d'éducation sous-estiment le nombre d'étudiants temporairement mobiles).

- Certains jeunes qui ont suivi une formation à l'étranger avant de retourner dans leur pays d'origine pour leurs études. Par définition, ces étudiants dont la mobilité est évidente, appelés «étudiants mobiles vers leur pays d'origine », ne sont par représentés dans les statistiques sur les étudiants étrangers.

L'ampleur de ces différences n'est visible que pour quelques pays européens qui recueillent des données à la fois sur la nationalité et la mobilité des étudiants et qui se caractérisent par de fortes proportions d'étudiants mobiles souhaitant étudier chez eux. Dans tous ces pays, plus de $20 \%$ des étudiants étrangers ont suivi une formation dans le pays d'études avant le début des études, et entre $4 \%$ et $15 \%$ des étudiants mobiles sont allés (ou souvent, retournés) dans leur pays d'origine pour suivre leurs études.

En 2006, l'Academic Cooperation Association (Kelo, Teichler and Wächter 2006, pp. 15-16) a démontré que pour 32 pays européens :

- parmi les 19,4 millions d'étudiants en 2003 (année universitaire 2002/2003), environ 1,1 million étaient de nationalité étrangère, soit 5,8 \% ;

- la nationalité de près de la moitié des étudiants étrangers, soit 2,9\% de l'ensemble des étudiants, était celle d'un autre pays européen ;

- environ 575000 étudiants ont étudié à l'étranger, soit 3,1\% des étudiants ayant étudié dans leur pays d'origine ;

- environ $82 \%$ des étudiants européens qui étudient à l'étranger (soit 2,5\% de l'ensemble des étudiants européens) sont allés dans un autre pays européen. 


\section{Encadré 1 \\ Les différentes enquêtes utilisées}

Cet article s'appuie sur les données de plusieurs enquêtes:

Dans l'enquête CHEERS, plus de 35000 diplômés de l'année universitaire 1994/95 issus de 11 pays européens et du Japon ont été interrogés en 1999, soit quatre ans environ après l'obtention de leur diplôme (Schomburg et Teichler, 2006). Au printemps 2000, une enquête de suivi a été réalisée dans le cadre de l'évaluation du programme SOCRATES 2000 auprès des diplômés, issus de cinq pays, qui avaient indiqué en 1999 avoir étudié à l'étranger pendant leurs études (Jahr et Teichler, 2002).

L'enquête REFLEX a été réalisée en 2005 auprès des diplômés de l'année universitaire 1999/2000 dans 12 pays européens et au Japon (cinq ans après l'obtention de leur diplôme). Plus de 40000 diplômés ont répondu à un long questionnaire qui comportait environ 400 variables.

Plusieurs études sur la transition entre l'enseignement supérieur et l'emploi et les premières années de vie active des anciens étudiants ERASMUS ont été menées par des membres du centre INCHER-Kassel (plus connu sous son ancien nom "Centre for Research on Higher Education and Work ") :

(1) ERASMUS 1992: au printemps 1992, plus de 1300 anciens étudiants ERASMUS de l'année universitaire 1988/99 ont fourni des informations sur une période d'environ trois ans après leur séjour ERASMUS à l'étranger (Teichler et Maiworm, 1994).

(2) ERASMUS 1994 : au printemps 1994, plus de 1200 anciens étudiants ERASMUS de l'année universitaire 1988/89 ont fourni des informations sur une période d'environ cinq ans après leur séjour ERASMUS à l'étranger (Maiworm et Teichler, 1996).

Ces deux études faisaient partie d'une étude longitudinale. Les anciens diplômés interrogés sur leurs études avaient déjà été interrogés au cours de l'année universitaire 1989/90, peu de temps après leur séjour ERASMUS à l'étranger.

(3) Le projet VALERA (valeur de la mobilité ERASMUS) vise à définir l'impact de la mobilité dans le cadre du sous-programme ERASMUS de SOCRATES sur les carrières des étudiants et enseignants mobiles. En 2005, des enquêtes représentatives ont été réalisées dans ce but auprès d'anciens étudiants mobiles ERASMUS (4 600 personnes interrogées), employeurs (312 personnes interrogées), responsables d'universités (626) et anciens enseignants mobiles ERASMUS (755) (Bracht et al., 2006).

Les données statistiques recueillies régulièrement par l'UNESCO montrent que le nombre d'étudiants qui étudient dans un pays différent de celui de leur nationalité a augmenté mais que, proportionnellement, le nombre d'étudiants étrangers dans le monde entier est resté plus ou moins stable, à $2 \%$ près. La mobilité des étudiants est généralement «verticale», c'est-à-dire de pays plus pauvres vers des pays relativement plus riches et d'universités de niveau moins élevé vers des établissements d'un niveau a priori plus élevé (voir Teichler, 2004). L’UNESCO (1998) a publié un aperçu des développements apportés à l'enseignement supérieur en 1998, année de la
Déclaration de la Sorbonne', à l'occasion de la Conférence mondiale sur l'enseignement supérieur de l'UNESCO. Il montrait un taux d'augmentation irrégulier du nombre d'étudiants étrangers dans les principaux pays d'accueil européens entre le milieu

\footnotetext{
${ }^{1}$ Cette Déclaration de la Sorbonne sur «l'harmonisation de l'architecture du système européen d'enseignement supérieur »a été signée à l'université de la Sorbonne de Paris, en mai 1998, par les ministres chargés de l'Enseignement supérieur français, italien, britannique et allemand. Un suivi de cette mesure a été assuré à Bologne le 19 juin 1999, où 29 ministres européens chargés de l'enseignement supérieur ont signé une déclaration qui vise à créer un Espace européen de l'enseignement supérieur d'ici à 2010 (la Déclaration de Bologne).
} 
des années 80 et des années 90, autour d'un pourcentage annuel moyen de $5 \%$. Des taux d'augmentation annuels supérieurs à $10 \%$ ont été relevés pour le Royaume Uni, le Portugal, la Hongrie, la Finlande et le Danemark, et entre 5 et $10 \%$ dans la plupart des autres pays. Au contraire, le nombre d'étudiants étrangers a diminué au cours de la même période en Grèce, en République tchèque, en Italie et, dans une moindre mesure, en France.

En 2005, l'OCDE (2005, p. 267) a présenté un aperçu de l'évolution du nombre d'étudiants étrangers entre 1998 et 2003. Sur la moyenne des 16 pays européens qui ont transmis leurs données pour les deux années, la part d'étudiants étrangers a augmenté de $5,2 \%$ à $6,7 \%$ en cinq ans.

Pour ce qui est de la mobilité étudiante temporaire, les statistiques de la Commission européenne révèlent que le nombre d'étudiants ERASMUS est passé de 97571 en 1997/98 à 123897 en 2002/03. Cette augmentation de $27 \%$ en cinq ans est en réalité légèrement inférieure à celle de l'ensemble des étudiants étrangers dans les pays européens au cours de la même période. Sur la base des diverses informations, nous estimons qu'environ 250000 à 300000 étudiants européens ont été temporairement mobiles en 2003.

L'étude fournit une image plus détaillée de la mobilité internationale. Par exemple, il est possible d'établir le taux de participation à la mobilité étudiante temporaire en demandant aux étudiants qui passent leur diplôme dans leur pays d'origine de fournir cette information peu avant ou après les épreuves.

L'enquête REFLEX, meilleure source de données disponible en la matière, a traité le sujet de la mobilité internationale à différents stades de la vie des diplômés ( $c f$. tableau 1).

Environ $5 \%$ des diplômés interrogés dans l'enquête REFLEX sont nés dans un autre pays que celui de l'établissement d'enseignement supérieur, et l'on peut supposer que la plupart d'entre eux sont des citoyens étrangers. $11 \%$ des diplômés revendiquent un passé migratoire - nés à l'étranger, ou de parents nés à l'étranger - avec des différences importantes selon les pays. En Suisse, un diplômé sur trois a un passé migratoire et on observe également des pour- centages élevés au Royaume-Uni (23\%), en France (16\%) et en Autriche (15\%). Environ $2 \%$ des personnes interrogées vivaient dans un pays différent de celui d'obtention du diplôme à 16 ans. En fait, la plupart des diplômés interrogés qui viennent d'un autre pays ont déménagé dans le pays de leurs études lorsqu'ils étaient enfants ou pendant leur scolarité.

La mobilité internationale pendant les études est signalée par $26 \%$ des diplômés. En fait, $21 \%$ ont séjourné à l'étranger pour leurs études et $7 \%$ ont travaillé à l'étranger pendant leurs études.

Aussi, environ $2 \%$ ont à la fois séjourné et travaillé à l'étranger pendant leurs études. D'autres enquêtes auprès des étudiants et diplômés montrent que la plupart des étudiants ayant indiqué avoir travaillé à l'étranger ont en fait réalisé un stage, c'est-à-dire qu'ils ont acquis une expérience professionnelle dans le cadre de leurs études.

Le Tableau 1 montre des différences importantes au niveau de la mobilité pendant les études selon le pays d'obtention du diplôme.

En fait, ceux qui se rendent à l'étranger pendant leurs études pour étudier ou travailler séjournent dans l'autre pays en moyenne sept mois pour les études et six mois pour le travail. Ces données extraites de l'enquête REFLEX confirment globalement les résultats d'autres études disponibles sur la mobilité étudiante temporaire en Europe.

Parmi les personnes interrogées dans les pays d'Europe occidentale pour l'enquête CHEERS, qui ont été diplômées il y a quatre ans environ, $18 \%$ ont séjourné à l'étranger pendant leurs études pour étudier ou pour travailler (Jahr et Teichler, 2007), soit environ deux tiers des personnes ayant fourni les mêmes informations pour l'enquête REFLEX. Les données disponibles mettent en évidence le fait que la mobilité étudiante temporaire était en hausse dans les années 90 en Europe, fait également observé par d'autres enquêtes réalisées auprès d'étudiants.

$7 \%$ des diplômés autour de l'année 2000, interrogés dans l'enquête REFLEX et diplômés dans leur pays d'origine, ont indiqué avoir séjourné dans un autre pays au cours des cinq années suivant l'obtention $d u$ 


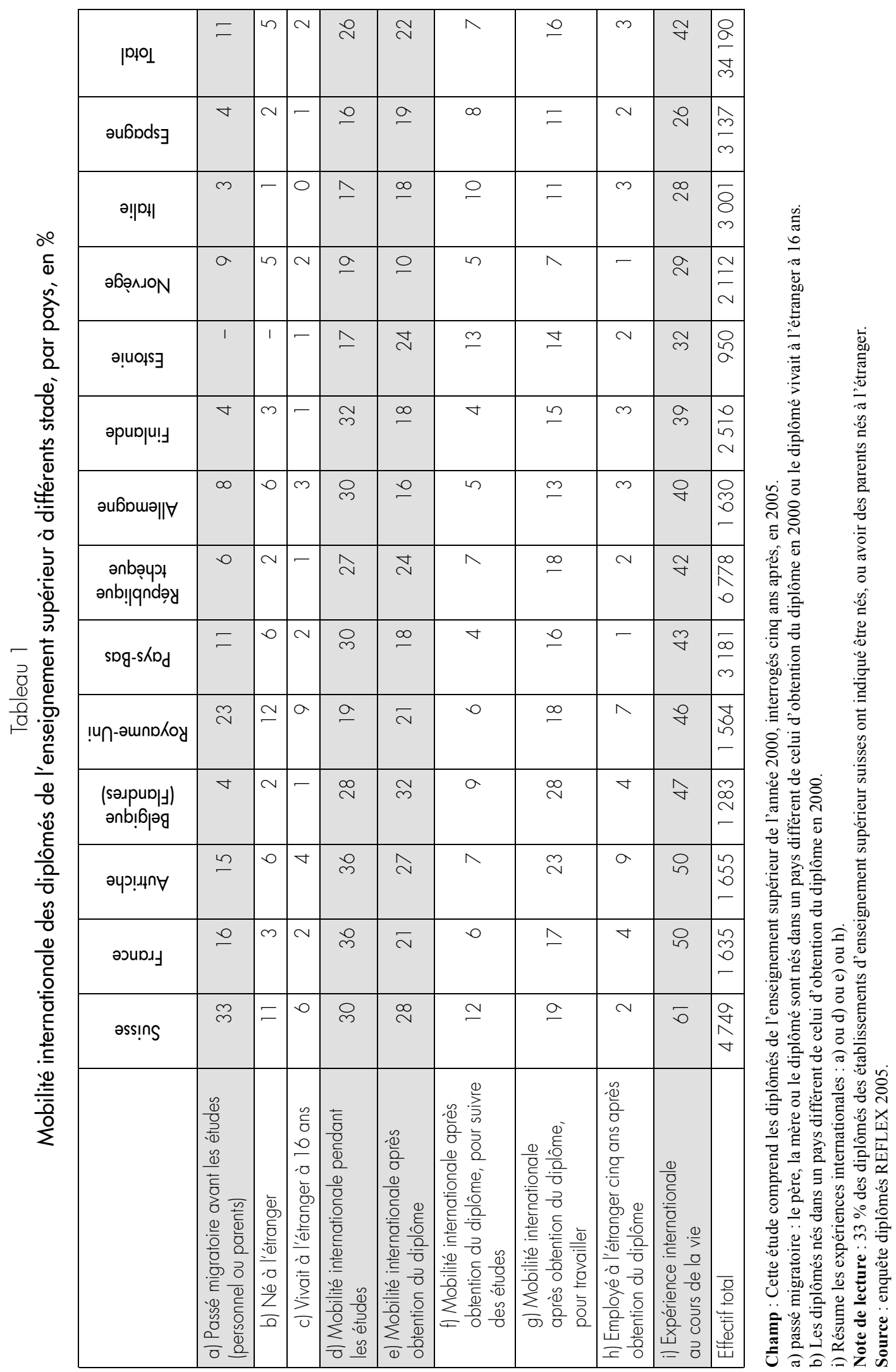


diplôme pour poursuivre leurs études. Cette catégorie présente le pourcentage le plus important chez les diplômés d'Estonie (13\%) et de Suisse (12\%). Dans la mesure où $40 \%$ des personnes interrogées ont entamé d'autres études au cours des cinq années qui ont suivi leur diplôme, nous notons qu'environ un sixième de ces diplômés ont étudié en partie dans un autre pays. La durée moyenne des études suivies après l'obtention du diplôme à l'étranger est de quatre mois. Cela confirme les résultats des autres études selon lesquels l'expérience à l'étranger pendant leurs études motive les étudiants à poursuivre leurs études à un niveau avancé de qualification (voir Bracht et al., 2006).

Parmi les personnes interrogées pour l'enquête CHEERS et diplômées quatre ans auparavant, environ $3 \%$ avaient un emploi à l'étranger au début de leur carrière professionnelle, occupaient un emploi et vivaient à l'étranger au moment de l'enquête. $18 \%$ d'entre eux ont indiqué avoir travaillé à l'étranger au cours des quatre années suivant l'obtention de leur diplôme, dont la plupart sont mandatés par leur employeur dans leur pays natal (Jahr et Teichler, 2007). Les divergences entre les résultats des enquêtes CHEERS et REFLEX sont trop faibles pour être significatives d'une augmentation au cours de la période de cinq ans.

En associant les différents modes d'expériences internationales (passé migratoire avant les études, études temporaires à l'étranger, mobilité internationale au cours des cinq années suivant l'obtention du diplôme), nous remarquons, comme l'indique le Tableau 1, que l'expérience internationale dans son ensemble, jusqu'à cinq ans après l'obtention du diplôme, désignée ici par "expérience internationale au cours de la vie », est répartie dans différents pays européens, et notamment en Suisse (61\%), en France et en Autriche (50\% chacun), alors qu'elle est plus rare en Norvège, Italie et Espagne (moins de $30 \%$ ).

Des statistiques sur le marché du travail suggèrent que seulement $3 \%$ environ de la main-d'œuvre hautement qualifiée en Europe viennent des autres pays européens. Les résultats des enquêtes CHEERS et REFLEX semblent correspondre à ces données et n'indiquent pas de tendance claire à la croissance.
Parmi tous les diplômés qui travaillent à l'étranger cinq ans après l'obtention de leur diplôme et interrogés pour l'enquête REFLEX, $17 \%$ ont choisi l'Allemagne, $12 \%$ le Royaume-Uni, $11 \%$ la Suisse et $9 \%$ les États-Unis.

\section{Comme l'illustre le Tableau 2 :}

- l'Allemagne s'avère la destination préférée des diplômés autrichiens ( $43 \%$ des diplômés des universités d'Autriche travaillant à l'étranger) et néerlandais $(32 \%)$;

- des préférences similaires peuvent être observées pour les diplômés français qui vont en Suisse (31\%), pour les diplômés belges qui vont aux Pays-Bas (33\%), les tchèques qui vont au Royaume-Uni (34 \%), et les norvégiens qui vont en Suède (34\%) et aux États-Unis (35\%), tandis que les diplômés d'autres pays ont choisi des destinations plus diversifiées.

Les diplômés étrangers (nés à l'étranger) présentent des schémas très différents de mobilité internationale après l'obtention du diplôme par rapport à ceux qui sont nés dans le pays d'obtention. Parmi les diplômés de l'étude REFLEX nés dans un pays différent de celui de l'obtention de leur diplôme et qui travaillent, $16 \%$ étaient employés dans leur pays natal et $5 \%$ étaient employés dans un autre pays environ cinq ans après l'obtention du diplôme. Logiquement, de nombreuses personnes qui obtiennent leur diplôme à l'étranger, contrairement à celles qui l'obtiennent dans leur pays d'origine, ne sont pas intéressées uniquement par une carrière dans le pays où elles ont obtenu leur diplôme mais plutôt par la poursuite de cette mobilité internationale.

\section{L'IMPACT DE LA MOBILITÉ INTERNATIONALE SUR LES DÉBUTS DE VIE ACTIVE}

L'impact de la mobilité internationale pendant les études sur la carrière des diplômés peut être analysé d'après les deux enquêtes CHEERS et REFLEX, en comparant les personnes qui ont été mobiles pendant leurs études à celles qui ne l'ont pas été. Les données nous permettent de suivre les conséquences des choix possibles d'un point de vue social et 
Tableau 2

Principales destinations pour le travail à l'étranger, cinq ans après l'obtention du diplôme (en \%)

\begin{tabular}{|c|c|c|c|c|c|c|c|c|c|c|c|c|c|}
\hline $\begin{array}{r}\text { Pays } \\
\text { d'études }\end{array}$ & 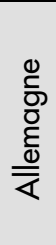 & 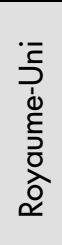 & 岕 & $\begin{array}{l}\text { ơ } \\
\dot{0} \\
\dot{\hat{\partial}} \\
0\end{array}$ & 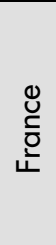 & 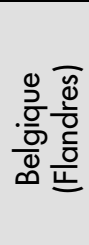 & 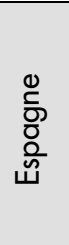 & $\frac{. \underline{\Xi}}{\underline{\underline{\underline{D}}}}$ & $\frac{0}{\frac{U}{\frac{U}{3}}}$ & 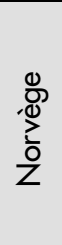 & $\frac{\frac{0}{0}}{\frac{\mathrm{C}}{0}}$ & 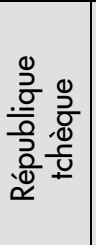 & $\begin{array}{l}\overline{0} \\
0\end{array}$ \\
\hline Allemagne & - & 2 & 22 & 32 & 16 & 7 & 12 & 9 & 43 & 0 & 12 & 20 & 17 \\
\hline Royaume-Uni & 10 & - & 21 & 7 & 5 & 21 & 17 & 16 & 4 & 9 & 12 & 34 & 12 \\
\hline Suisse & 16 & 6 & - & 0 & 31 & 0 & - & 9 & 15 & 0 & 8 & 3 & 11 \\
\hline États-Unis & 18 & 15 & 16 & 12 & 0 & 7 & 16 & 9 & 6 & 35 & 4 & 4 & 9 \\
\hline Pays-Bas & 2 & 0 & 7 & - & 3 & 33 & 2 & 0 & 3 & 10 & 6 & 6 & 7 \\
\hline France & 6 & 13 & 15 & 2 & - & 16 & 13 & 9 & 2 & 0 & 0 & 2 & 6 \\
\hline Belgique (Flandres) & 5 & 2 & 5 & 11 & 5 & - & 6 & 6 & 3 & 0 & 4 & 8 & 4 \\
\hline Luxembourg & 15 & 0 & 0 & 0 & 13 & 2 & 2 & 0 & 1 & 0 & 0 & 0 & 4 \\
\hline Suède & 3 & 0 & 0 & 4 & 0 & 0 & 0 & 3 & 0 & 34 & 23 & 1 & 3 \\
\hline Espagne & 2 & 0 & 3 & 0 & 4 & 2 & - & 10 & 0 & 0 & 6 & 1 & 3 \\
\hline Irlande & 0 & 12 & 0 & 4 & 2 & 0 & 6 & 3 & 0 & 6 & 7 & 0 & 2 \\
\hline Canada & 3 & 3 & 0 & 3 & 8 & 0 & 2 & 3 & 0 & 0 & 0 & 3 & 2 \\
\hline Italie & 0 & 7 & 5 & 0 & 0 & 0 & 8 & - & 3 & 0 & 0 & 1 & 2 \\
\hline Danemark & 0 & 0 & 0 & 0 & 0 & 0 & 1 & 5 & 1 & 0 & 9 & 0 & 1 \\
\hline Chine & 2 & 2 & 0 & 0 & 3 & 0 & 0 & 2 & 1 & 0 & 0 & 0 & 1 \\
\hline Liechtenstein & 0 & 0 & 3 & 0 & 0 & 0 & 0 & 0 & 5 & 0 & 0 & 0 & 1 \\
\hline Autriche & 3 & 0 & 2 & 0 & 0 & 0 & 2 & 3 & - & 0 & 4 & 1 & 1 \\
\hline Norvège & 0 & 6 & 0 & 0 & 0 & 0 & 3 & 0 & 0 & - & 4 & 0 & 1 \\
\hline Australie & 0 & 5 & 0 & 3 & 0 & 0 & 0 & 0 & 2 & 0 & 0 & 0 & 1 \\
\hline Émirats Arabes Unis & 0 & 0 & 0 & 0 & 0 & 2 & 0 & 0 & 2 & 0 & 0 & 0 & 1 \\
\hline Russie & 0 & 3 & 0 & 3 & 0 & 0 & 0 & 1 & 1 & 0 & 0 & 1 & 1 \\
\hline Thailande & 6 & 0 & 0 & 4 & 0 & 0 & 0 & 0 & 0 & 0 & 0 & 0 & 1 \\
\hline Slovaquie & 0 & 0 & 0 & 0 & 0 & 0 & 0 & 0 & 0 & 0 & 0 & 10 & 1 \\
\hline Roumanie & 0 & 0 & 0 & 0 & 3 & 0 & 0 & 0 & 1 & 0 & 0 & 0 & 1 \\
\hline Afrique du Sud & 0 & 0 & 0 & 0 & 0 & 0 & 0 & 0 & 3 & 0 & 0 & 0 & 1 \\
\hline Portugal & 0 & 0 & 0 & 0 & 0 & 0 & 8 & 0 & 0 & 7 & 0 & 0 & 1 \\
\hline Autres & 10 & 24 & 0 & 15 & 5 & 10 & 2 & 13 & 7 & 0 & 2 & 5 & 8 \\
\hline
\end{tabular}

Champ : diplômés dans le pays d'études.

Note de lecture : $31 \%$ des diplômés mobiles issus des établissements d'enseignement supérieur français sont allés travaillés en Suisse. Le pourcentage total ne correspond pas toujours à $100 \%$ en raison de difficultés d'arrondi.

Source : Enquête diplômés REFLEX 2005.

biographique ainsi qu'en fonction des différents aspects des profils d'études. En outre, l'analyse peut s'appuyer sur deux études d'évaluation portant sur l'impact d'une étude temporaire réalisée dans le cadre du programme ERASMUS (Maiworm \& Teichler, 1996 ; Bracht et al., 2006). 
Le Tableau 3 présente quelques résultats tirés de l'enquête REFLEX relatifs aux différences observées sur les profils sociaux et biographiques de formation entre les personnes qui ont été mobiles au cours de leurs études et/ou peu après l'obtention de leur diplôme et les personnes qui n'ont pas été mobiles à ce moment de leur vie. Celles qui ont été mobiles :

- sont issues plus souvent de familles dont les parents sont diplômés de l'enseignement supérieur ;

- ont souvent suivi des études supérieures conduisant à des études doctorales (programmes de master ou programmes universitaires longs en formation initiale);

- sont plus souvent engagées dans des organisations étudiantes ou bénévoles ;
- ont plus souvent effectué des stages ou acquis d'autres expériences professionnelles pendant leurs études.

En outre, il faut noter que les étudiants mobiles ont été largement surestimés dans les filières de lettres et surestimés pour moins de la moitié dans les filières de l'éducation ainsi que de la santé et du bien-être ; tandis que les femmes sont sous-représentées.

Les résultats de l'enquête REFLEX sur les diplômés confirment les résultats précédents (Jahr \& Teichler, 2002), c'est-à-dire que la mobilité internationale pendant ou peu après les études ne semble pas améliorer les carrières professionnelles (voir Tableau 4). On observe quelques légères différences seulement concernant par exemple le statut professionnel en général, l'utilisation des connaissances sur un emploi ou la satisfaction professionnelle.

Tableau 3

Mobilité internationale et caractéristiques personnelles et de formation, en \%

\begin{tabular}{|c|c|c|c|c|c|}
\hline & \multicolumn{4}{|c|}{ Mobilité internationale } & \multirow[b]{2}{*}{ Total } \\
\hline & $\begin{array}{l}\text { Aucune } \\
\text { mobilité }\end{array}$ & $\begin{array}{l}\text { Pendant les } \\
\text { études } \\
\text { uniquement }\end{array}$ & $\begin{array}{l}\text { Après les } \\
\text { études } \\
\text { uniquement }\end{array}$ & $\begin{array}{l}\text { Pendant et } \\
\text { après les } \\
\text { études }\end{array}$ & \\
\hline \multicolumn{6}{|l|}{ Sexe } \\
\hline Femmes & 61 & 63 & 47 & 53 & 59 \\
\hline \multicolumn{6}{|l|}{ Plus haut niveau d'éducation du père } \\
\hline Enseignement supérieur (CITE $5+6)$ & 38 & 49 & 44 & 51 & 42 \\
\hline Type de programme d'études & & & & & \\
\hline $\begin{array}{l}\text { Programme long conduisant directement } \\
\text { - à un titre de chercheur de haut niveau (CITE 5A) }\end{array}$ & 49 & 64 & 56 & 68 & 54 \\
\hline \multicolumn{6}{|l|}{$\begin{array}{l}\text { Expérience professionnelle au cours des études } \\
\text { supérieures }\end{array}$} \\
\hline $\begin{array}{l}\text { Expérience professionnelle liée aux études } \\
\text { au cours des études supérieures }\end{array}$ & 44 & 53 & 44 & 54 & 46 \\
\hline $\begin{array}{l}\text { Expérience professionnelle indépendante } \\
\text { des études au cours des études supérieures }\end{array}$ & 47 & 58 & 53 & 57 & 50 \\
\hline Effectif & $(15042)$ & (3 598) & (2 594) & (2 379) & $(23613)$ \\
\hline
\end{tabular}

Champ : diplômés de l'année universitaire 1999-2000 dans 12 pays européens et au Japon interrogés 5 ans après l'obtention de leur diplôme. CITE $=$ Classification Internationale Type de l'Éducation. Les niveaux 5 et 6 de la CITE désignent le niveau des diplômes d'enseignement supérieur.

Note de lecture : parmi les étudiants n'ayant eu aucune mobilité, $38 \%$ ont un père ayant suivi un parcours universitaire, contre $42 \%$ parmi l'ensemble.

Source : Enquête diplômés REFLEX 2005. 
Tableau 4

Mobilité internationale selon le type d'emploi occupé cinq ans après l'obtention du diplôme, en \%

\begin{tabular}{|l|c|c|c|c|}
\hline & Aucune & $\begin{array}{c}\text { Pendant les } \\
\text { études } \\
\text { uniquement }\end{array}$ & $\begin{array}{c}\text { Après les } \\
\text { études } \\
\text { uniquement }\end{array}$ & $\begin{array}{c}\text { Pendant et } \\
\text { après les } \\
\text { études }\end{array}$ \\
\hline Emploi permanent en 2005 & 80 & 77 & 77 & 73 \\
\hline Emploi à temps plein en 2005 (35+) & 78 & 80 & 83 & 80 \\
\hline Le niveau d'éducation correspond à l'emploi actuel & 84 & 86 & 87 & 86 \\
\hline Poste occupé * (ClTP 88 1 et 2) & 70 & 77 & 77 & 79 \\
\hline Forte utilisation des connaissances et des compétences & 73 & 72 & 72 & 76 \\
\hline Satisfaction professionnelle élevée & 69 & 68 & 71 & 70 \\
\hline
\end{tabular}

Champ : diplômés de l'année universitaire 1999-2000 dans 12 pays européens et au Japon interrogés 5 ans après l'obtention de leur diplôme. * CITP 88 Classification internationale type des professions : groupe $1:$ membres de l'exécutif et des corps législatifs, cadres supérieurs de l'administration publique, dirigeants et cadres supérieurs d'entreprise. Groupe $2:$ professions intellectuelles et scientifiques.

Source : Enquête diplômés REFLEX 2005.

Le Tableau 5 montre par exemple que ceux qui ont été mobiles à l'international perçoivent, dans la plupart des pays, un salaire légèrement plus élevé. Les diplômés mobiles masculins de Suisse, du RoyaumeUni et d'Estonie gagnent plutôt moins que les diplômés masculins non mobiles de ces mêmes pays. Dans la plupart des pays, les femmes mobiles ont un avantage salarial supérieur aux hommes mobiles. En France par exemple, les femmes mobiles gagnent $27 \%$ de plus que les femmes diplômées non mobiles.

Tableau 5

Mobilité internationale et variation de revenu mensuel en euro par pays et par sexe

\begin{tabular}{|l|c|c|c|c|c|c|}
\hline & \multicolumn{3}{|c|}{ Hommes } & \multicolumn{3}{c|}{ Femmes } \\
\hline & Non mobiles & Mobiles & $\begin{array}{c}\text { Variation } \\
\text { salariale en \% }\end{array}$ & Non mobiles & Mobiles & $\begin{array}{c}\text { Variation } \\
\text { salariale en \% }\end{array}$ \\
\hline Suisse & 4636 & 4585 & -1 & 3957 & 3901 & -1 \\
\hline France & 2463 & 3021 & 23 & 1924 & 2439 & 27 \\
\hline Autriche & 3177 & 3283 & 3 & 2419 & 2690 & 11 \\
\hline Belgique (Flandres) & 2839 & 2886 & 2 & 2510 & 2614 & 4 \\
\hline Royaume-Uni & 3127 & 3036 & -3 & 2568 & 2824 & 10 \\
\hline Pays-Bas & 2718 & 2853 & 5 & 2307 & 2449 & 6 \\
\hline République tchèque & 922 & 1034 & 12 & 775 & 885 & 14 \\
\hline Allemagne & 4221 & 4939 & 17 & 3248 & 3778 & 16 \\
\hline Finlande & 2984 & 3092 & 4 & 2311 & 2481 & 7 \\
\hline Estonie & 1195 & 1189 & -1 & 855 & 881 & 3 \\
\hline Norvège & 4171 & 4720 & 13 & 3280 & 3703 & 13 \\
\hline Italie & 1840 & 2205 & 20 & 1509 & 1662 & 10 \\
\hline Espagne & 1733 & 1862 & 7 & 1429 & 1605 & 12 \\
\hline
\end{tabular}

Source : Enquête diplômés REFLEX 2005.

Champ : diplômés de l'année universitaire 1999-2000 dans 12 pays européens et au Japon interrogés 5 ans après l'obtention de leur diplôme. Moyenne arithmétique; diplômés employés à temps plein.

Note de lecture : les hommes diplômés suisses non mobiles gagnent 4636 euros 5 ans après l'obtention de leur diplôme, contre 4585 pour les mobiles. 
Plusieurs analyses de régression multiple ont été réalisées séparément par pays et par type de programmes d'études (voir note du Tableau 6) dans les pays qui disposent de variables de passé social et biographique et d'analyse du profil d'études. Comme le Tableau 6 l'indique, la mobilité pendant et/ou peu après les études a un faible impact sur les salaires si les facteurs sociaux, biographiques et d'éducation sont contrôlés, ce qui devrait favoriser l'emploi et le travail. Mais ces résultats ne sont pas homogènes pour tous les pays, comme en témoignent les chiffres sur la Suisse, les Pays-Bas, le Royaume-Uni.

Tableau 6

Impact sur les revenus de la mobilité internationale pendant et après les études, par pays et par type de programme d'études (coefficients de régression; $M C O$ )

\begin{tabular}{|c|c|c|c|}
\hline Pays & $\begin{array}{l}\text { Type de } \\
\text { programme } \\
\text { d'études }\end{array}$ & $\begin{array}{l}\text { Variable dépendante : } \\
\text { mobilité internationale } \\
\text { pendant les études }\end{array}$ & $\begin{array}{c}\text { Variable dépendante: } \\
\text { mobilité internationale } \\
\text { pendant et/ou peu après les études }\end{array}$ \\
\hline \multirow[t]{2}{*}{ Suisse } & Type 1 & & 232 \\
\hline & Type 2 & & \\
\hline \multirow[t]{2}{*}{ France } & Type 1 & & 196 \\
\hline & Type 2 & 350 & 477 \\
\hline \multirow[t]{2}{*}{ Autriche } & Type 1 & & \\
\hline & Type 2 & & 183 \\
\hline \multirow[t]{2}{*}{ Belgique (Flandres) } & Type 1 & 226 & \\
\hline & Type 2 & & \\
\hline \multirow[t]{2}{*}{ Royaume-Uni } & Type 1 & & \\
\hline & Type 2 & & -697 \\
\hline \multirow[t]{2}{*}{ République tchèque } & Type 1 & & \\
\hline & Type 2 & 76 & 82 \\
\hline \multirow[t]{2}{*}{ Allemagne } & Type 1 & 1207 & 1015 \\
\hline & Type 2 & & \\
\hline \multirow[t]{2}{*}{ Finlande } & Type 1 & & 161 \\
\hline & Type 2 & & \\
\hline \multirow[t]{2}{*}{ Norvège } & Type 1 & 429 & 336 \\
\hline & Type 2 & & \\
\hline \multirow[t]{2}{*}{ Italie } & Type 1 & & \\
\hline & Type 2 & 223 & 259 \\
\hline \multirow[t]{2}{*}{ Espagne } & Type 1 & & 183 \\
\hline & Type 2 & 226 & 128 \\
\hline
\end{tabular}

Champ : diplômés de l'année universitaire 1999/2000 dans 12 pays européens et au Japon (cinq ans après l'obtention de leur diplôme). Type 1 : Programmes d'études au niveau CITE 5A, ne conduisant pas directement à un titre de chercheur de haut niveau (comme Fachhochschule en Allemagne ou HBO aux Pays-Bas). Type 2 : Programmes d'études de niveau CITE 5A, conduisant à un titre de chercheur de haut niveau.

Note de lecture : en France, on observe un avantage d'environ 477 euros sur le salaire brut mensuel des étudiants mobiles diplômés des grandes Écoles ou qui ont obtenu un DEA ou DESS (diplôme d'études approfondies et diplôme d'études supérieures spécialisées), par rapport aux diplômés non mobiles pendant leurs études.

Résultats significatifs au seuil de $5 \%$. Les résultats ne sont pas significatifs pour l'Estonie.

Source : Enquête diplômés REFLEX 2005. 
Le tableau 6 présente les résultats de l'analyse de régression multiple avec deux variables dépendantes : a) la mobilité internationale pendant les études ; b) la mobilité internationale pendant et/ou peu après les études. Les variables de passé social et biographique et d'analyse du profil d'études ont été étudiées.

\section{L'IMPACT DE LA MOBILITÉ SUR LE TYPE D'ACTIVITÉ PROFESSIONNELLE}

La mobilité internationale semble influencer fortement le type d'activité professionnelle. Comme le Tableau 7 l'indique, les diplômés mobiles sont plus souvent employés dans des grandes entreprises. Ils sont bien plus souvent engagés dans des organismes à dimension internationale.

Les anciens étudiants ERASMUS interrogés pour l'enquête VALERA considéraient que les compé- tences suivantes acquises à l'international étaient importantes dans leur emploi actuel: ainsi, la connaissance professionnelle des autres pays était importante pour $45 \%$ d'entre eux ; la connaissance et la compréhension des différences internationales culturelles et sociales pour $57 \%$; le travail avec des personnes de milieux culturels différents pour $66 \%$ et la communication en langues étrangères pour $69 \%$.

Ces pourcentages sont de $4 \%$ à $10 \%$ supérieurs à ceux des anciens étudiants ERASMUS diplômés en 1994/95 qui ont été interrogés pour l'enquête CHEERS. Cela indique que les compétences internationales sont de plus en plus importantes pour les anciens étudiants ERASMUS. L'enquête réalisée auprès des diplômés de 1994/95 a montré, comme on pouvait s'y attendre, que ces compétences internationales étaient substantiellement plus importantes pour les diplômés qui ont été mobiles pendant leurs études que pour ceux qui ne l'ont pas été.

\section{Tableau 7}

Mobilité internationale et organisme employeur cinq ans après l'obtention du diplôme, en \%

\begin{tabular}{|l|c|c|c|c|}
\hline & $\begin{array}{c}\text { Aucune } \\
\text { mobilité }\end{array}$ & $\begin{array}{c}\text { Pendant } \\
\text { les études } \\
\text { uniquement }\end{array}$ & $\begin{array}{c}\text { Après } \\
\text { les études } \\
\text { uniquement }\end{array}$ & $\begin{array}{c}\text { Pendant } \\
\text { et après } \\
\text { les études }\end{array}$ \\
\hline $\begin{array}{l}\text { Taille de l'organisme } \\
\text { 1-49 }\end{array}$ & 35 & 29 & 28 & 32 \\
$50-999$ & 38 & 41 & 37 & 39 \\
1 Oo0+ & 27 & 30 & 35 & 29 \\
\hline Portée de l'activité de l'organisme & & & & \\
Locale & 24 & 15 & 11 & 11 \\
Régionale & 26 & 25 & 15 & 16 \\
Nationale & 24 & 25 & 21 & 21 \\
Internationale & 26 & 35 & 53 & 52 \\
\hline
\end{tabular}

Champ : les diplômés qui vivent, cinq après l'obtention de leur diplôme, dans leur pays d'origine.

Note de lecture : parmi les étudiants n'ayant connu aucune mobilité, $35 \%$ travaillent dans un organisme de moins de 50 salariés.

Source : Enquête diplômés REFLEX. 


\section{LE RECUL DE LA VALEUR PROFESSIONNELLE DES ÉTUDES À L'ÉTRANGER ?}

Cependant, toutes les informations disponibles indiquent que les nouveaux diplômés mobiles pendant leurs études mentionnent un recul de l'adéquation de leur expérience internationale au niveau professionnel par rapport aux cohortes d'étudiants qui ont séjourné à l'étranger avant eux. Il en est de même pour l'aspect général de l'emploi et du travail. Comme le montre le Tableau 8, le pourcentage d'anciens étudiants ERASMUS qui pensent que des études temporaires à l'étranger leur ont permis d'obtenir leur premier emploi a chuté de $71 \%$ à $54 \%$ en 12 ans. De même, le pourcentage de ceux qui voient un impact sur le type de tâches qu'ils accomplissent a diminué de $49 \%$ à $39 \%$, et enfin le pourcentage de ceux qui considèrent que les études temporaires à l'étranger ont permis d'améliorer leur niveau de salaire a diminué de $25 \%$ à $16 \%$.

Les anciens étudiants ERASMUS ont également observé un léger recul de l'utilisation, au niveau professionnel, de compétences visibles à l'international. Selon le Tableau 9, cet aspect est plus prononcé pour l'usage des langues étrangères que pour l'utilisation des connaissances sur le pays d'accueil.

Tableau 8

Perception de l'influence positive des études ERASMUS sur l'emploi et le travail, en \%

\begin{tabular}{|l|c|c|c|}
\hline & $\begin{array}{c}\text { Étudiants 1988/89 } \\
\text { ERASMUS } \\
\text { interrogés en 1993 }\end{array}$ & $\begin{array}{c}\text { Diplômés } \\
1994 / 95 \\
\text { interrogés en 1999 }\end{array}$ & $\begin{array}{c}\text { Étudiants 2000/01 } \\
\text { ERASMUS } \\
\text { interrogés en 2005 }\end{array}$ \\
\hline Obtention du premier emploi & 71 & 66 & 54 \\
\hline Type de tâches professionnelles & 49 & 44 & 39 \\
\hline Niveau de salaire & 25 & 22 & 16 \\
\hline
\end{tabular}

Champ : les diplômés ayant un emploi.

Note de lecture : $71 \%$ des étudiants ERASMUS 1988-89 considèrent que le programme ERASMUS a favorisé l'obtention de leur premier emploi.

Source : Enquête VALERA.

Tableau 9

Connaissances sur les pays d'accueil des étudiants ERASMUS, en \%

\begin{tabular}{|l|c|c|c|}
\hline & $\begin{array}{c}\text { Étudiants 1988/89 } \\
\text { ERASMUS } \\
\text { interrogés en 1993 }\end{array}$ & $\begin{array}{c}\text { Diplômés } \\
\text { 1994/95 } \\
\text { interrogés en 1999 }\end{array}$ & $\begin{array}{c}\text { Étudiants 2000/01 } \\
\text { ERASMUS } \\
\text { interrogés en 2005 }\end{array}$ \\
\hline Langue du pays d'accueil parlée & 47 & 42 & 38 \\
\hline Langue du pays d'accueil lue et écrite & 47 & 40 & 38 \\
\hline $\begin{array}{l}\text { Utilisation de ses propres connaissances } \\
\text { professionnelles sur le pays d'accueil }\end{array}$ & 30 & 25 & 25 \\
\hline $\begin{array}{l}\text { Utilisation de ses propres connaissances } \\
\text { sur le pays d'accueil au niveau culture/social }\end{array}$ & 30 & 32 & 24 \\
\hline Déplacements professionnels vers le pays d'accueil & 17 & 18 & 14 \\
\hline
\end{tabular}

Champ : diplômés ayant un emploi.

Note de lecture : parmi les étudiants ERASMUS 88/89, $47 \%$ parlent la langue du pays d'accueil.

Source : Enquête VALERA. 
En outre, les employeurs interrogés en 2006 (dans le cadre de l'enquête VALERA) ont exprimé un point de vue plus positif sur la valeur professionnelle qu'ils accordent à la mobilité internationale. Pour plus de 40 \% d'entre eux, les diplômés ayant une expérience internationale sont plus enclins à accepter des missions professionnelles avec de fortes responsabilités. $21 \%$ pensent que les diplômés ayant une expérience internationale peuvent espérer, après quelques années, un salaire plus élevé que ceux qui n'ont pas eu cette expérience (Bracht et al., 2006).

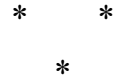

Les enquêtes réalisées quelques années après l'obtention du diplôme et qui analysent rétrospectivement les parcours d'éducation et les carrières des personnes interrogées révèlent la place importante de la mobilité à l'étranger. Parmi les étudiants diplômés autour de l'année 2000 , plus de $20 \%$ ont séjourné à l'étranger pendant leurs études et plus de $40 \%$ ont eu une expérience internationale quelconque, que ce soit le passé migratoire de leurs parents ou des périodes d'études supérieures ou de travail après l'obtention de leur diplôme.

Les informations fournies entre autres par l'étude REFLEX indiquent que les personnes ayant acquis une expérience internationale avant d'obtenir leur diplôme, ou peu de temps après, sont nettement plus enclines à la mobilité internationale et à accepter des emplois qui requièrent des compétences internationales dans le pays d'origine. Cela confirme une forte relation «horizontale» entre l'expérience et les études à l'international et le travail à l'échelle internationale. Les expériences internationales semblent conduire à des emplois dans des organismes à dimension internationale dans lesquels les compétences internationales sont reconnues.

Il existe également des relations « verticales », c'està-dire entre l'expérience internationale et la réussite professionnelle en général, relations moins étroites et moins significatives. D'une certaine manière, le travail à l'étranger et le travail qui nécessite des compétences à l'international bénéficient d'une reconnaissance en termes de statut et de tâches professionnelles. De même, les études temporaires à l'étranger semblent procurer certains avantages de carrière.

Mais ces avantages «verticaux » sont plus rares et moins importants. Il en va différemment selon les domaines : les carrières internationales nécessitent de longues périodes de transition vers l'emploi et sont souvent liées à des risques professionnels plus importants. De plus, certains avantages visibles ne sont qu'illusion, car les carrières internationales et les exigences professionnelles à l'international sont plus souvent orientées vers les secteurs économiques et les catégories de poste qui offrent un statut supérieur à la moyenne. Enfin, parmi ceux qui possèdent une expérience internationale antérieure à leurs études, certains sont des étudiants migrants offrant des avantages inégalés dans leur pays d'études et de travail.

Mais globalement, l'acquisition de compétences et d'expériences internationales ainsi que le choix d'un emploi qui nécessite des compétences internationales et probablement une mobilité professionnelle à l'international sont en moyenne mieux reconnus que d'autres choix d'études et de carrières.

La situation évolue ces dernières années. Le nombre d'étudiants et de diplômés ayant une expérience à l'international augmente et, à certains égards, les nouvelles générations mobiles sont bien plus compétentes dans des domaines visibles liés aux différentes expériences internationales. Cependant, une part plus faible des récentes cohortes d'anciens étudiants mobiles note un réel impact de leurs expériences internationales sur leurs carrières en général et sur la dimension internationale de leurs tâches professionnelles. Moins les expériences internationales sont sélectives, moins elles créent de différences. Les compétences internationales, le travail à l'étranger et les activités professionnelles visibles à l'international sont en augmentation, mais il est nécessaire que la préparation à ces activités et à ce travail se diversifie au-delà de la « voie royale » de la mobilité étudiante. 
Bracht O., Engel C., Janson K., Over A., Schomburg H. \& Teichler U. (2006), The professional value of Erasmus Mobility, Final Report, Presented to the European Commission - DG Education and Culture. Kassel: INCHER, University of Kassel 2006 (PdfDatei, online November 2006. URL: http:// www.ec.europa.eu/education/programmes/socrates/ erasmus/evalcareer.pdf)

Burn B., Cerych L. \& Smith A. (Eds.) (1990), Study abroad programmes, London: Jessica Kingsley Publishers.

Institute for Statistics (2006), Global Education Digest 2006, Montreal: UNESCO Institute for Statistics.

Jahr V. \& Teichler U. (2002), «Employment and work of former mobile students ", in Teichler U. (Ed.), ERASMUS in the SOCRATES programme: Findings of an evaluation study, pp. 117-135, Bonn: Lemmens.

Maiworm F. \& Teichler U. (1996), Study abroad and early career: experiences of former ERASMUS students, London and Bristol, PA: Jessica Kingsley Publishers.

OECD (2005), Education at a Glance: OECD Indicators 2004, Paris, OECD.

Opper S., Teichler U. \& Carlson J. (1990), The impact of study abroad programmes on students and graduates, London: Jessica Kingsley Publishers.

Rosselle D. \& Lentiez A. (1999), The ERASMUS programme 1987-1995: Looking to the future, vol. 2, Lille: Pôle Universitaire Europeén.
Schomburg H. (2007), « The Professional Success of Higher Education Graduates », in European Journal of Education, Vol. 42, $\mathrm{n}^{\circ} 1$.

Schomburg H. \& Teichler U. (2006), Higher Education and Graduate Employment in Europe. Results of Graduates Surveys from 12 Countries, Dordrecht: Springer, Higher Education Dynamics.

Teichler U. (2007) (Ed.), Careers of University Graduates. Views and Experiences in Comparative Perspective, Dordrecht: Springer, Higher Education Dynamics.

Teichler U. (2004), « The Changing Debate on Internationalisation of Higher Education », Higher Education, vol. 48, n ${ }^{\circ}$, pp. 5-26.

Teichler U. (Ed.). (2002), ERASMUS in the SOCRATES programme: Findings of an evaluation study, Bonn: Lemmens.

Teichler U. \& Maiworm F. (1997), The ERASMUS experience: Major findings of the ERASMUS evaluation research project, Luxembourg: Office for Official Publications of the European Communities.

Teichler U. \& Maiworm F. (1994), Transition to Work: The Experience of Former ERASMUS Students. London and Bristol, PA: J. Kingsley Publishers.

UNESCO (1998), World Statistical Outlook on Higher Education: 1980-1995, Paris: UNESCO.

Wissenschaft weltoffen (2006), Hannover: HIS Hochschulinformationssystem $\mathrm{GmbH}$. 


\title{
Résumé
}

\section{Mobilité internationale des étudiants et débuts de vie active}

\author{
Harald Schomburg et Ulrich Teichler
}

Les enquêtes réalisées quelques années après l'obtention du diplôme et qui analysent a posteriori les parcours scolaires et sociaux des jeunes interrogés montrent une importante mobilité vers l'étranger. Les informations fournies par l'étude REFLEX et d'autres études indiquent que les personnes ayant acquis une expérience internationale avant ou peu après l'obtention de leur diplôme sont beaucoup plus enclines à la mobilité internationale et à accepter des emplois qui exigent des compétences internationales dans leur pays d'origine. Cela confirme une forte corrélation entre les études et l'expérience à l'international d'une part et le travail à l'échelle internationale d'autre part.

\section{Mots clés}

Fuite des cerveaux, Mobilité internationale, Formation initiale, Insertion professionnelle, Enquête, Europe Journal of Economic Literature : F 22, J 61, J 24 